\title{
Increased CCL19 and CCL21 levels promote fibroblast ossification in ankylosing spondylitis hip ligament tissue
}

Yang Qin ${ }^{1,2+}, \mathrm{Li} \mathrm{Da} \mathrm{He}^{1 \dagger}$, Zhou Jian Sheng ${ }^{3 \dagger}$, Miao Ming Yong ${ }^{3}$, Yang Sheng Sheng ${ }^{3}$, Xu Wei Dong ${ }^{1 *}$, Tong Wen Wen ${ }^{1}$ and Zou Yu Ming ${ }^{1}$

\begin{abstract}
Background: It is well-documented that both chemokine (C-C motif) ligand 19 (CCL19) and 21 (CCL21) mediate cell migration and angiogenesis in many diseases. However, these ligands' precise pathological role in ankylosing spondylitis (AS) has not been elucidated. The objective of this study was to examine the expression of CCL19 and CCL21 (CCL19/CCL21) in AS hip ligament tissue (LT) and determine their pathological functions.

Methods: The expression levels of CCL19, CCL21 and their receptor CCR7 in AS ( $n=31)$ and osteoarthritis (OA, $n=21) L T$ were analyzed via real-time polymerase chain reaction (RT-PCR) and immunohistochemistry (IHC). The expression of CCL19, CCL21 and CCR7 in AS ligament fibroblasts was also detected. The proliferation of ligament fibroblasts was measured via a cell counting kit-8 (CCK8) assay after exogenous CCL19/CCL21 treatment. Additionally, the role of CCL19/CCL21 in osteogenesis was evaluated via RT-PCR and enzyme-linked immunosorbent assay (ELISA) in individual AS fibroblast cultures. Furthermore, the expression of the bone markers alkaline phosphatase (ALP), osteocalcin (OCN), collagenase I (COL1), integrin-binding sialoprotein (IBSP) and the key regulators runt-related transcription factor-2 (Runx-2) and osterix were investigated. Moreover, the CCL19/CCL21 levels in serum and LT were measured via ELISA.

Results: The mRNA levels of CCL19/CCL21 in AS hip LT were significantly higher than that in OA LT, and IHC analysis revealed a similar result. Exogenous CCL19/CCL21 treatment did not affect the proliferation of ligament fibroblasts but significantly up-regulated the expression of bone markers, including ALP and OCN, and the key regulators Runx-2 and osterix. In addition, the serum levels of CCL19/CCL21 were apparently elevated in AS patients compared to healthy controls $(\mathrm{HC})$, and the expression of the two chemokines correlated significantly in AS patients.
\end{abstract}

Conclusions: CCL19 and CCL21, two chemokines displaying significantly associated expression in serum, indicating a synergistic effect on AS pathogenesis, may function as promoters of ligament ossification in AS patients.

Keywords: CCL19, CCL21, Ankylosing spondylitis, Fibroblast, Ossification

\section{Background}

Ankylosing spondylitis (AS) is the representative rheumatic disease of seronegative spondyloarthropathy and is characterized primarily by recurrent inflammatory back pain and bilateral sacroiliitis [1]. Hip ankylosis, which is typically accompanied by enthesis heterotopic ossification

\footnotetext{
*Correspondence: xuwdshanghai@126.com

${ }^{\dagger}$ Equal contributors

'Department of Orthopedics, Changhai Hospital Affiliated to the Second Military Medical University, Changhai Road 168, Shanghai 200433, Yangpu district, P. R. China

Full list of author information is available at the end of the article
}

(HO), occurs in about one-third of patients suffering from AS [2]. Entheopathy in peripheral and axial sites induces distinct pathologic changes, and inflammatory enthesitis is clinically detectable in approximately $10 \%$ of patients suffering from early-stage AS and 50\% of those suffering from established AS [3]. Fibroblasts are the most numerous connective tissue cells in enthesis or ligament tissue (LT) and are reported to be associated with heterotopic ossification (HO) in LT $[4,5]$. However, the relevant mechanism by which fibroblasts mediate $\mathrm{HO}$ remains unclear. 
CCL19, CCL21 and their corresponding receptor CCR7 are described to be crucially involved in the adaptive immune system, their primary role being the migration of monocytes/macrophages, mature dendritic cells (DCs) and naive $\mathrm{T}$ cells to lymph nodes [6-9]. Studies have demonstrated that CCR7 is responsible for the migration of T cells into inflamed tissues and T-cell egress from these tissues via the afferent lymph under inflammatory conditions [10] and that CCR7 knockout attenuates the development of diseases such as coronary sclerosis [11]. Moreover, CCR7 signaling is considered to mediate both angiogenesis and tumor metastasis in different tumor microenvironments [12-14].

The effects of these two chemokines on proliferation, adhesin and/or integrin avidity, invasiveness, endocytosis, differentiation and survival have been comprehensively summarized in a prospective review [15].

Numerous studies have revealed their respective roles in immune diseases, such as rheumatoid arthritis (RA), eosinophilic pneumonia, breast cancer and acquired immune deficiency syndrome (AIDS) [16-18]. A recent study demonstrated that CCL19 was more strongly expressed in systemic sclerosis (SSc) skin and was correlated to vascular inflammation [19], providing further evidence for the role of CCL19 in perivascular inflammation and immune cell recruitment. A parallel study illustrated that serum CCL19 levels might reflect blood B cell imbalance and correlate to the levels of some serum B cell biomarkers, such as rheumatoid factor, anti-CCP, free light chains, IgG, IgA and IgM, in RA patients. Furthermore, serum CCL19 also acted as a potential predictor of the clinical response to rituximab in B cell-mediated RA subtypes [20]. All of these results illustrate the pivotal function of CCL19 in clinical settings. However, the role of CCL19/ CCL21 in ankylosing spondylitis is rarely reported and demands a more comprehensive understanding.

In this study, we preliminarily focus on AS patients experiencing severely limited hip activity (average BASFI = 62.24, which means severe functional limitations). First, we detected higher expression of CCL19, CCL21 in LT and ligament fibroblasts. No significant effect on LT fibroblast proliferation was detected upon exposure to these two chemokines. Next, we examined the roles of CCL19/ CCL21 in the osteogenic potential of AS ligament fibroblasts. The expression of the bone markers ALP, OCN, Runx-2 and Osterix were up-regulated by CCL19/21 simulation. However, IBSP and COL1 expression was not seen up-regulated. Serum CCL19/CCL21 levels are closely associated, and CCL19 expression appeared to correlate to the Visual Analogue Scale (VAS) Pain Score and disease duration, whereas CCL21 expression did not display this correlation. Our result reveals a novel role of CCL19/ CCL21 in ligament ossification and may provide useful evidence for further investigation in AS patients.

\section{Methods}

\section{Patients}

Patients meeting the modified New York criteria [21] for AS and patients suffering from single hip OA who underwent total hip arthroplasty at our department from May 1, 2012, to October 31, 2013, were selected for this study. The demographic and clinical characteristics of all the subjects are summarized in Table 1. All patients who provided their written informed consent according to the Declaration of Shanghai Changhai Hospital were enrolled. This study was approved by Shanghai Changhai Hospital Ethics Committee and the Ethics Committee approval document number is CHEC2013-194 The LT samples were stored in a $-80^{\circ} \mathrm{C}$ freezer after collection in the operating room. Primary cultures of ligament fibroblasts were generated according to a previously described method [22] with some modifications.

\section{RNA extraction and real-time PCR}

The LT was fully shredded using RNase-free scissors and transferred to a $1.5 \mathrm{ml}$ RNase-free EP tube. A total of $1 \mathrm{ml}$ of TRIzol (Invitrogen) was used for no more than $100 \mathrm{mg}$ of LT. TRIzol was used according to the manufacturer's instructions. Total RNA was reverse-transcribed to cDNA using a ReverTra Ace qPCR RT Kit (TOYOBO CO., LTD., Osaka, Japan). The transcripts were stored at $-80^{\circ} \mathrm{C}$ until further use. For RT-PCR, SYBR Green Real Time PCR Master Mix (TOYOBO CO., LTD., Osaka, Japan) was used in a total volume of $20 \mu \mathrm{L}$. The primer sequences are listed in Table 2. The samples were evaluated in triplicate using an equal load of $10 \mathrm{ng}$ of cDNA/well, and three independent experiments were performed. The data were collected using an ABI 7300 Real-Time PCR System.

\section{Immunohistochemistry}

The LT samples were immediately fixed using $4 \%$ formaldehyde and embedded in paraffin. The samples were deparaffinized in xylene for 20 minutes at room temperature, followed by rehydration using an alcohol gradient. Antigens were unmasked by first incubating the slides in $0.01 \mathrm{~mol} / \mathrm{L}$ boiling citrate buffer at $\mathrm{pH} 6.0$ for 15 minutes and then cooling at room temperature. Endogenous peroxidase activity was blocked via incubation in $3 \% \mathrm{H}_{2} \mathrm{O}_{2}$ for 5 minutes, followed by blocking with goat serum for 30 minutes at $37^{\circ} \mathrm{C}$. The primary antibody rabbit anti-human CCL19 (1:100, R\&D Systems), rabbit anti-CCL21 (1:100, R\&D Systems), rabbit anti-CCR7 (1:100, ABGENT), or an isotype-matched irrelevant antibody (R\&D Systems) was individually added to the sections at the proper concentration. Following three washes with PBS, the slides were incubated for $30 \mathrm{~min}$ in the secondary antibody (GT Vision III anti-mouse/rabbit Universal immunohistochemical 
Table 1 Demographic characteristics of AS and OA patients and healthy controls

\begin{tabular}{|c|c|c|c|}
\hline & Ankylosing spondylitis (AS) $(n=44)$ & Osteoarthritis (OA) $(n=29)$ & Healthy controls $(H C)(n=16)$ \\
\hline Male/female & $35 / 7$ & $20 / 9$ & $9 / 7$ \\
\hline Age (y) & $35.93 \pm 11.30$ & $43.48 \pm 11.21$ & $33.38 \pm 5.88$ \\
\hline HLA-B27 (+) & 36 & NA & NA \\
\hline $\mathrm{ESR}(\mathrm{mm} / \mathrm{h})$ & $42.29 \pm 25.74$ & $8.92 \pm 8.26$ & NA \\
\hline CRP (mg/L) & $25.41 \pm 19.75$ & $9.56 \pm 21.96$ & NA \\
\hline BASDAI score & $52.3 \pm 14.7$ & NA & NA \\
\hline BASFI score & $62.24 \pm 17.98$ & NA & NA \\
\hline VAS score & $5.26 \pm 1.94$ & NA & NA \\
\hline BAS-G score & $5.51 \pm 1.56$ & NA & NA \\
\hline Disease duration (months) & $155 \pm 103$ & NA & NA \\
\hline Morning stiffness (min) & $13 \pm 16$ & NA & NA \\
\hline Serum CCL19 (pg/ml) & $354.12 \pm 75.49$ & $304.55 \pm 82.82$ & $215.15 \pm 50.20$ \\
\hline Serum CCL21 (pg/ml) & $535.33 \pm 124.22$ & $470.13 \pm 79.75$ & $390.92 \pm 38.12$ \\
\hline
\end{tabular}

The data are expressed as the means \pm Standard deviation (SD). ESR: Erythrocyte sedimentation rate; CRP: C-reactive protein; BASDAl: Bath Ankylosing Spondylitis Disease Activity Index; BASFI: Bath Ankylosing Spondylitis Functional Index; VAS: Visual analog scale; BAS-G: Bath Ankylosing Spondylitis Patient Global Score. NA: Not applicable.

detection kit, Dako, Denmark) at $37^{\circ} \mathrm{C}$. The slides were washed 3 times with PBS and then stained with DAB for 5 minutes and hematoxylin for $45 \mathrm{~s}$, followed by rinsing with tap water for 5 minutes. Finally, the slides were processed via alcohol gradient dehydration, xylene immersion and resin blocking. Three fields from each slide were randomly selected, and tissue staining was analyzed using Image-Pro Plus 6.0 software. The scored data were pooled, and the mean density value \pm standard deviation (SD) was calculated for each group.

\section{Primary cell isolation, culture, and procedures}

The previously described method for primary cell isolation was mentioned performed [22] with some modifications. The LT from patients suffering from AS or OA who underwent hip joint replacement surgery was washed with sterile saline to remove the blood. Then, the fats and synovial tissue were cleared from the LT, followed by shredding into $1-2 \mathrm{~mm}^{3}$ tissue blocks. The tissue blocks were centrifuged twice in $15 \mathrm{ml}$ centrifuge tubes in $10 \mathrm{ml}$ of PBS at $1000 \mathrm{r} / \mathrm{min}$. Next, the tissue blocks were digested in $5 \mathrm{ml}$ of PBS and $500 \mu \mathrm{l}$ of collagenase I (Sigma-Aldrich China, Shanghai, China) for $5 \mathrm{~h}$ at $37^{\circ} \mathrm{C}$. Then, $5 \mathrm{ml}$ of $0.25 \%$ trypsin was added, and the samples were mixed and incubated at $37^{\circ} \mathrm{C}$ for 2 minutes. The samples were filtered using 200 mesh filters, and the filtrate was centrifuged at $1000 \mathrm{r} / \mathrm{m}$ for 5 minutes. The supernatant was discarded. Then, $5 \mathrm{ml}$ of DMEM/ high glucose culture medium supplemented with $100 \mathrm{U} / \mathrm{ml}$ penicillin (Invitrogen, Carlsbad, California, USA), $100 \mu \mathrm{g} / \mathrm{ml}$

Table 2 Primer sequences used for real-time PCR

\begin{tabular}{|c|c|c|}
\hline & Sense primer ( $5^{\prime}$ to $\left.3^{\prime}\right)$ & Anti-sense primer $\left(5^{\prime}\right.$ to $\left.3^{\prime}\right)$ \\
\hline Human CCL19 & CCAACTCTGAGTGGCACCAA & TGAACACTACAGCAGGCACC \\
\hline Human CCL21 & TGGCCTCTTACTCACCCTCT & GCCTCTTGATCCCCTTAGCC \\
\hline Human CCR7 & GCCTACGACGTCACCTACAG & GGCAGAAGAGTCGCCTATGG \\
\hline Beta-actin & CCATCGTCCACCGCAAAT & TGTCACCTTCACCGTTCC \\
\hline ALP & ATCTTGGTCTGGCTCCCATG & TTTCCCGTTCACCGTCCAC \\
\hline Human OCN & GGCAGCGAGGTAGTGAAGAG & CTGGAGAGGAGCAGAACTGG \\
\hline Human COL1 & GAG AGC ATG ACC GAT GG & GTG ACG CTG TAG GTG AA \\
\hline IBSP & CGCCAATGAATACGACAATG & GATGCAAAGCCAGAATGGAT \\
\hline Human VEGF-A & GAGCCTTGCCTTGCTGCTCTA & CACCAGGGTCTCGATTGGAT \\
\hline Runx-2 & GCAGCAACCCAGAAACACTT & AACACATGACCCAGTGCAAA \\
\hline Osterix & AGAGGAGAGACTCGGGACAG & GAGTTGTTGAGTCCCGCAGA \\
\hline
\end{tabular}

CCL19: Chemokine (C-C motif) ligand 19; CCR7: Chemokine receptor 7; ALP: Alkaline phosphatase; OCN: Osteocalcin; COL1: Collagenase I; IBSP: Integrin-binding sialoprotein; VEGF-A: Vascular endothelial growth factor-A; Runx-2: Runt-related transcription factor-2. 
streptomycin (Invitrogen, Carlsbad, California, USA) and $12 \%$ FBS was added to the centrifuge tube. The samples were mixed by pipetting and transferred to culture flasks in a humidified $5 \% \mathrm{CO} 2$ incubator at $37^{\circ} \mathrm{C}$ The passages from 3 to 8 were used. For osteogenic differentiation, the fibroblasts were cultured to confluence and then incubated in DMEM/high glucose medium containing $100 \mathrm{nM}$ dexamethasone, $50 \mu \mathrm{g} / \mathrm{ml}$ ascorbic acid, $10 \mathrm{mM}$ of $\beta$-glycerophosphate $[23,24]$ and $12 \%$ fetal bovine serum.

\section{Flow cytometric analysis}

The expression of CD90 (Biolegend) was identified as a molecular marker of fibroblasts based on FACS analysis as previously described $[25,26]$. The fibroblasts were detached via brief incubation in $2 \mathrm{mM}$ EDTA in PBS. Following fixation using $1 \mathrm{ml}$ of fix buffer (0.5\% BSA-PBS) and incubation at room temperature for $10 \mathrm{~min}$, approximately $1 \times 10^{6}$ cells were stained at room temperature via incubation in an anti-CCR7 pAb (polyclonal antibody, catalog \# AP4998a, ABGENT) or an irrelevant IgG2a isotype control at a concentration of $0.025 / \mathrm{ml}$ for $90 \mathrm{~min}$. After two washing and centrifugation steps, the cells were incubated in a PE-conjugated goat-anti-rabbit IgG pab (Tianjin Sungene Biotech Co., Ltd, Tianjin, China) for $40 \mathrm{~min}$. Then, the cells were immediately analyzed via flow cytometry, and the data were calculated using a FACSCalibur flow cytometer and CellQuest software (BD Biosciences).

\section{Measurement of fibroblast proliferation}

The proliferation of fibroblasts was examined via a cell counting kit-8 (CCK-8 kit) assay according to the manufacturer's instructions. Briefly, approximately $2 \times 10^{3}$ cells were seeded in a volume of $100 \mu \mathrm{l}$ DMEM on each well of a 96-well plate. A range of concentrations of CCL19/ CCL21 $(0-200 \mathrm{ng} / \mathrm{ml}$, Propetech) was added to the medium, and the cells were cultured for $48 \mathrm{~h}$. Mesangial (M) cells, which display a proliferative response upon exposure to CCL19 [27], were kindly provided by Professor Mei Changlin (Shanghai Eastern Hepatobiliary Hospital) as a positive control. Then, $100 \mu \mathrm{l}$ of fresh medium in $10 \mu \mathrm{l}$ of the CCK-8 solution was added to each well and incubated at $37^{\circ} \mathrm{C}$ for $2 \mathrm{~h}$. The absorbance (A) at $490 \mathrm{~nm}$ was measured. All assays were performed in quadruplicate, and three individual experiments were performed. The data are expressed as the mean values \pm SD of 4 wells per treatment.

\section{Assessment of fibroblast mineralization}

$1 \times 10^{6}$ fibroblasts from one AS LT were seeded on 6-well plates. Five groups were assigned: osteogenic differentiation medium as a positive control, CCL19 $(10 \mathrm{ng} / \mathrm{ml}$, according to previous studies [16] and the manufacturer's recommendations), CCL21 (10 ng/ml), CCL19/CCL21 (both $10 \mathrm{ng} / \mathrm{ml}$ ), and PBS as a negative control (Grow group). After culturing for $48 \mathrm{~h}$, total RNA was extracted to determine the mRNA expression levels of the osteogenesis-specific transcription factors Runx-2 and Osterix and the osteogenic differentiation markers IBSP, COL1, ALP and OCN. On days 2, 4 and 6, the culture supernatants were collected to detect of the levels of ALP and OCN (N-MID ELISA kit, Immuno-diagnostic Systems Inc, Fountain Hills, AZ, USA) via ELISA. All measurements were performed in duplicate.

\section{Statistical analysis}

All data are expressed as the means \pm standard deviation (SD). For statistical comparisons of two groups of samples, the Mann-Whitney U-test was used. Alternatively, comparisons of continuous variables were performed using Student's two-tailed t-test using SPSS 17.0 software; $\mathrm{p}<0.05$ was considered to be significant. The relative gene expression levels were determined according to the $2^{-\Delta \Delta} C_{t}$ method, and the results were expressed as the fold-change compared to the negative control.

\section{Results}

\section{Higher expression of CCL19 and CCL21 in AS LT than in} OA LT

We first investigated the expression of CCL19/CCL21 in AS and OA hip LT via real-time PCR and IHC. RT-PCR analysis revealed significantly increased transcriptional expression levels of CCL19/CCL21 (Figure 1A) in AS LT compared to OA LT $(\mathrm{P}=0.034$ and $\mathrm{P}=0.004)$. The smaller the $\Delta \mathrm{Ct}$ value, the higher the mRNA expression level. The IHC results further validated the higher expression of these two chemokines in the AS group than the OA group (Figure $1 \mathrm{~B}-\mathrm{C}, \mathrm{P}=0.016$ and $\mathrm{P}=0.023$ ). However, the expression their receptor CCR7 did not display a significant difference between the two groups (Figure 1A and $\mathrm{D}, \mathrm{P}=0.155$ and $\mathrm{P}=0.191$ ). Interestingly, we found that CCL19/CCL21 were primarily expressed on cells surrounding the capillaries, which likely corresponds to vascular endothelial cells, indicating a more important role in angiogenesis and migration of cells such as macrophages and dendritic cells. Previous studies found a proangiogenic effect of CCL19/CCL21 via the induction of secretion of vascular endothelial growth factor (VEGF) and angiotensin I (Ang-I) in fibroblasts [16]. Combined with our results, we propose that the expression of CCL19/ CCL21 in AS LT is involved in both inflammatory cell migration and abnormal angiogenesis.

\section{CCL19 and CCL21 does not exert a significant proliferative effect on ligament fibroblasts}

We next examined the expression of their receptor CCR7 on the ubiquitous LT fibroblasts. The fibroblasts in passages 

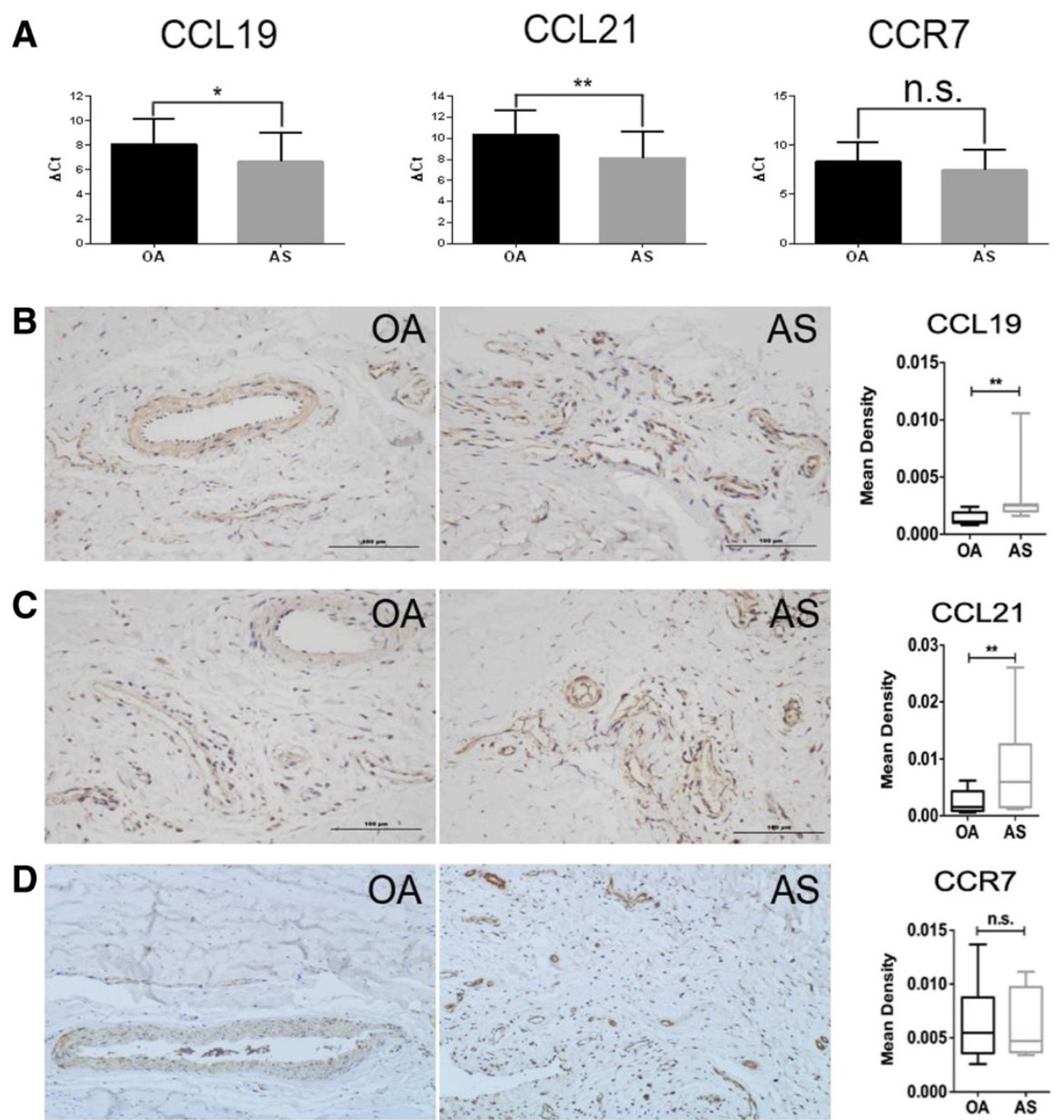

Figure 1 Expression of CCL19, CCL21 and CCR7 in AS and OA ligament tissue (LT). (A) RT-PCR analysis revealed the mRNA expression levels of CCL19, CCL21 and CCR7 in AS $(n=31) L T$ compared to OA $(n=19) L T(P=0.034,0.004$ and 0.155 , respectively). The data are expressed as the $\Delta C t$ value (Ct target gene-Ctßactin ) between the OA and AS groups, and the Mann-Whitney U-test was performed. (B-D) AS $(n=15)$ and OA $(\mathrm{n}=15) \mathrm{LT}$ were stained with rabbit anti-human CCL19 (B), rabbit anti-human CCL21 (C) or rabbit anti-human CCR7 (D), and the immunostaining was expressed as the mean density \pm standard deviation (SD). The expression levels of CCL19, CCL21 and CCR7 were compared between the AS and $\mathrm{OA}$ groups, with $\mathrm{P}$ values of $0.016,0.023$ and 0.191 , respectively. ${ }^{*} \mathrm{P}<0.05 ;{ }^{* *} \mathrm{P}<0.01 ; \mathrm{n} . \mathrm{s}$. $=$ no significance. The original magnification is $\times 200$ in $B, C$, and $D$.

3 to 8 were used to perform the expression and functional analyses. The fibroblasts from the AS and OA LT did not display any differences in morphology based on light microscopy (Figure 2A). FACS analysis revealed strong expression of the fibroblast marker CD90 but relatively weak expression of CCR7 (Figure 2B). However, RT-PCR and nucleic acid gel electrophoresis clearly confirmed the expression of CCL19, CCL21 and CCR7 in LT fibroblasts from AS $(n=3)$ and OA patients $(n=3)$ (Figure $2 \mathrm{C}$ ). These results indicated that increased levels of CCL19/CCL21 could stimulate LT fibroblast via CCR7 signaling. Moreover, we also detected secretion of CCL19 in the supernatants of 3 AS fibroblast cultures (Figure 2D), but CCL21 was not detected. This result implies that CCL19/CCL21 might be differently regulated in AS LT fibroblasts.

Because the abnormal proliferation of fibroblast-like cells was reported in ankle/tarsal joints of ankylosing enthesitis male mice [28] and CCL19/CCL21 induce proliferative effects on various cell types [15], we examined whether stimulation with CCL19/CCL21 for $48 \mathrm{~h}$ affected fibroblast proliferation in LT. As shown in Figure 2E, at various concentrations of CCL19/CCL21, the A490 value did not significantly differ, and only a limited proliferative effect was detected in both AS and OA LT fibroblasts ( $\mathrm{P}>0.05$, each compared to the Grow group). This result suggests that neither CCL19 nor CCL21 affected fibroblast growth. 

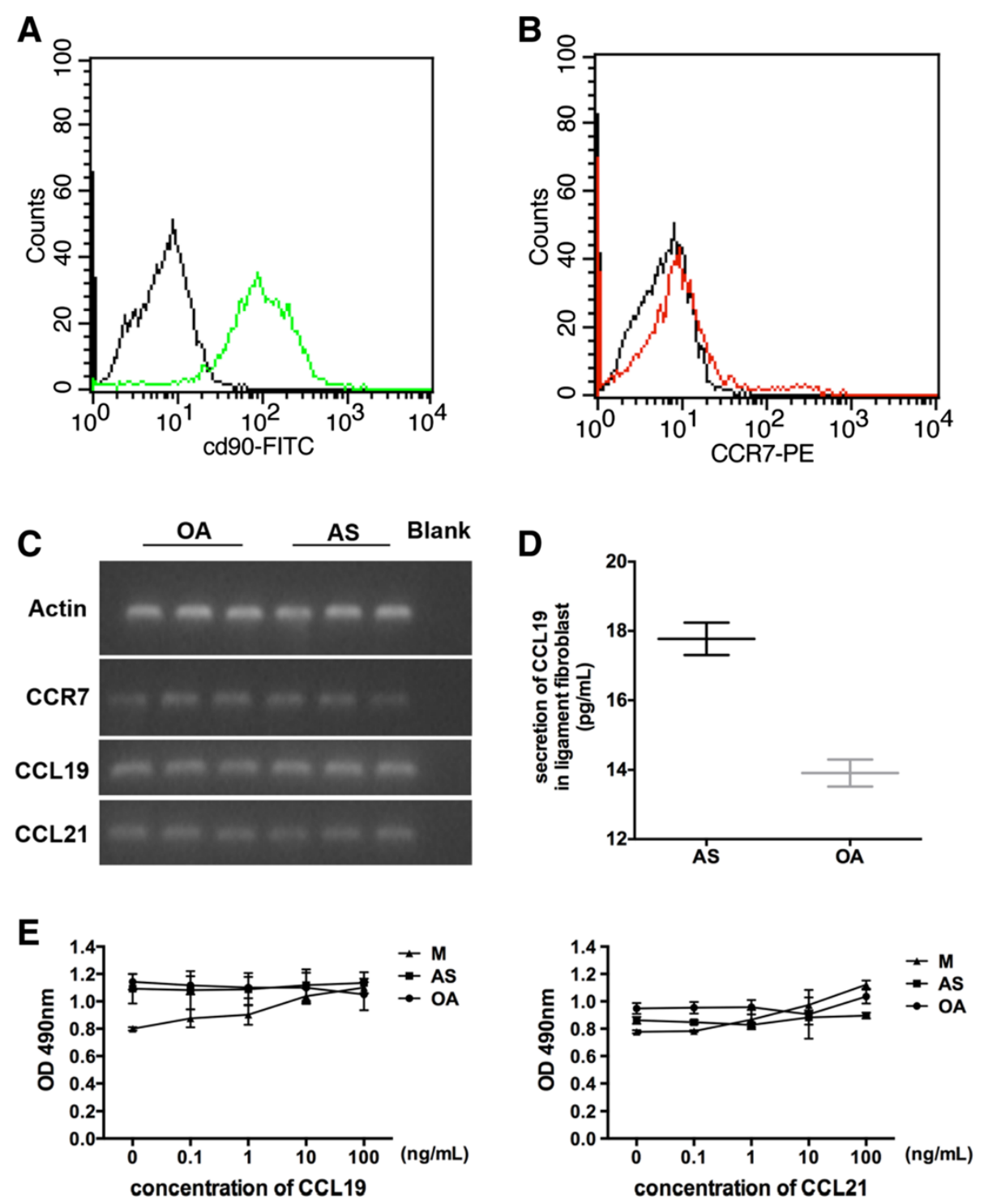

Figure 2 Expression levels of CCL19, CCL21 and CCR7 in fibroblasts and their role in fibroblast proliferation. (A) Morphology of in vitro cultured fibroblasts via light microscopy. The original magnification is $\times 100$. (B) A representative image of the expression of the fibroblast marker CD90 and CCR7 in AS LT fibroblasts. (C) RT-PCR and nucleic acid gel electrophoresis analyses revealed the expression of CCL19, CCL21 and CCR7 in $3 \mathrm{AS}$ and $3 \mathrm{OA}$ ligament fibroblast cultures. Blank corresponds to a control well containing no template cDNA. (D) ELISA revealed the secretion of CCL19 in 3 AS and 3 OA fibroblast cultures, and the experiments were repeated three times in duplicate. The data were calculated as the average value (CCL19 concentration) \pm Standard deviation (SD). (E) Incubation of fibroblasts in various concentrations of CCL19 and CCL21 for $48 \mathrm{~h}$. Cell proliferation was analyzed via the CCK8 assay as described in the Methods section. Cells cultured under standard growth conditions (growth medium) were used as the negative control. Additionally, mesangial (M) cells were used as the positive control. Changes in the proliferative activity were expressed as the values relative to the negative controls. Each result represents the mean \pm SD of 4 parallel incubations for each condition. Comparable results were obtained from three independent experiments.

\section{Promotion of osteogenesis in CCL19 and}

\section{CCL21-stimulated AS fibroblasts}

Inflammation and ligament ossification are common symptoms in AS patients. Here, we discovered the proosteogenic potential of CCL19/CCL21 in AS LT fibroblasts. Five groups were assigned: Grow, control, CCL19, CCL21 and CCL19/CCL21. The concentration of each chemokine was $10 \mathrm{ng} / \mathrm{ml}$. After treatment for $48 \mathrm{~h}$, ALP
mRNA expression was up-regulated more than 2-fold in the control, CCL19 and CCL19/CCL21 groups (Figure 3A, $\mathrm{P}=0.008, \mathrm{P}=0.026$ and $\mathrm{P}=0.0042$, respectively) compared to the Grow group, but the CCL21 group did not display a significant difference compared to the Grow group $(P=0.115)$. The ELISA results revealed increased secretion of ALP (Figure 3B) over the three time points (2, 4 and 6 days). However, two other bone markers, 

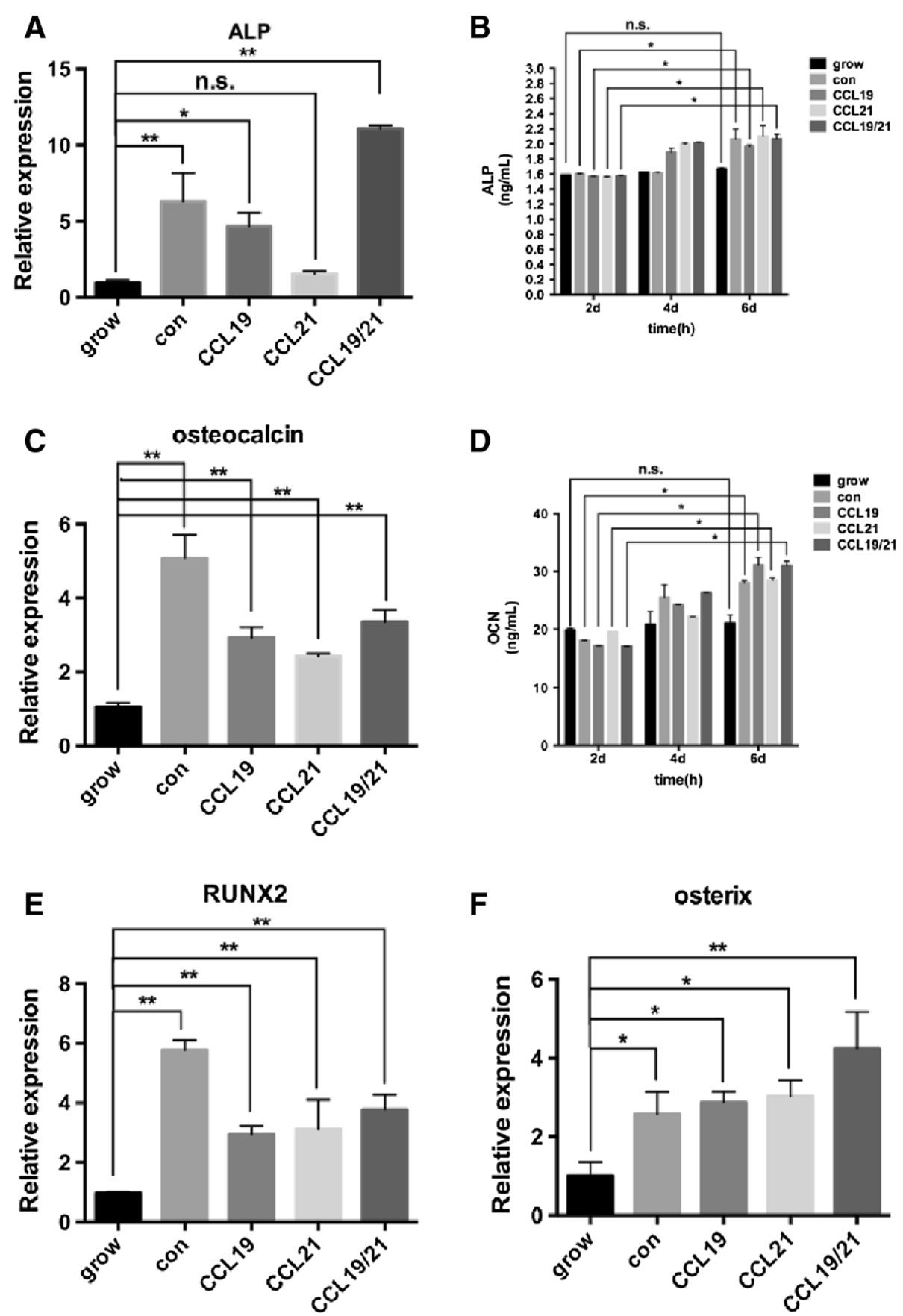

Figure 3 Effect of exogenous CCL19/CCL21 treatment on the osteogenic potential of AS LT fibroblasts after stimulation for various periods. Five groups were assigned: Grow (standard culture medium), control (osteogenesis-induced culture medium), CCL19 (standard culture medium supplemented with $10 \mathrm{ng} / \mathrm{ml}$ CCL19), CCL21 (standard culture medium supplemented with $10 \mathrm{ng} / \mathrm{ml}$ CCL21) and CCL19/21 (standard culture medium supplemented with $10 \mathrm{ng} / \mathrm{ml} \mathrm{CCL19}$ and $10 \mathrm{ng} / \mathrm{ml} \mathrm{CCL21).} \mathrm{ALL} \mathrm{data} \mathrm{are} \mathrm{compared} \mathrm{to} \mathrm{the} \mathrm{Grow} \mathrm{group,} \mathrm{and} \mathrm{the} \mathrm{unpaired} \mathrm{t-test}$ was performed. (A) Changes in the mRNA levels of the early bone marker ALP in AS fibroblasts after stimulation for $48 \mathrm{~h}$. The CCL19/CCL21 group displayed the maximal effect $(P<0.01)$, but the expression of CCL21 did not display a significant change. (B and D) ELISA revealed the secretion of ALP and OCN at various time points (2, 4 or 6 days). (C) Changes in the OCN mRNA levels after CCL19/CCL21 stimulation for 48 h. (E-F) The mRNA levels of the osteogenesis regulators Runx2 and osterix were also up-regulated by CCL19 or CCL21 treatment for $48 \mathrm{~h}$. ${ }^{* P}<0.05$; ${ }^{* * P}<0.01$; n.s. $=$ no significance. 
COL1 and IBSP, did not display this change (data not shown) in response to these two chemokines. The expression of the interim bone marker OCN demonstrated changes in both the mRNA and protein levels that were similar to those of ALP (Figure 3C-D). In addition, the expression of the key regulators Runx-2 and osterix, which modulate osteoblast differentiation, were also significantly up-regulated after CCL19/21 stimulation compared to the Grow group (Figure 3E-F). Because ALP and OCN are regulated by Runx-2 and osterix, these findings suggest that CCL19/CCL21 promote the ossification of AS ligament fibroblasts via Runx-2 and osterix.

\section{Clinical significance of CCL19 and CCL21}

The emerging role of CCL19 or CCL21 in the clinic has been identified for many diseases. Thus, we explored the possible relationship between CCL19/CCL21 and inflammation (based on ESR, CRP and the VAS score), function (BASFI), disease activity (BASDAI) and global assessment (BAS-G, disease duration, morning stiffness) in AS patients. The serum CCL19/CCL21 levels were significantly higher in AS patients than in OA patients and healthy controls $(\mathrm{HC})$ and were higher in OA patients than in $\mathrm{HC}$ (Figure 4A, both $\mathrm{P}<0.05$ compared to the OA or HC group). The CCL19/CCL21 levels were both higher in the AS LT compared to the OA LT (Figure 4B, each chemokine compared to the $\mathrm{OA}$ group, both $\mathrm{P}<0.01$ ). Correlation analysis revealed that the serum CCL19 level was closely associated with the serum CCL21 level $(\mathrm{r}=0.546, \mathrm{P}<0.01)$ and was also correlated with the VAS score $(r=0.327, P=0.035)$ and the disease duration $(\mathrm{r}=0.316, \mathrm{P}=0.041)$ (Table 3) in AS patients. No correlation was detected between the LT CCL19/CCL21 levels and any clinical indicator (data not shown). Furthermore, the serum CCL19/CCL21 levels were not correlated to the LT CCL19 or CCL21 levels in AS patients, which displayed a weak relationship between the different sites. These results reveal that the increased serum CCL19/ CCL21 levels are closely correlated and that CCL19 may be more influential in predicting global clinical manifestations in AS patients.

\section{Discussion}

In this study, we detected increased expression of CCL19/ CCL21 in AS hip LT. IHC staining of CCL19/CCL21, as well as their distribution, indicate that vascular endothelial cells and fibroblasts may represent the primary source of CCL19/CCL21. However, in vitro cultured AS ligament fibroblasts secreted only a small amount of CCL19 and no CCL21. This indicates that other cells, such as vascular endothelial cells, are likely the source of CCL19/CCL21. Moreover, inflammatory macrophages in enthesis and inflammatory factors, such as TNF- $\alpha$, LPS, which regulate the expression of CCL19/CCL21 in synovial fibroblasts [16], may also similarly mediate the production of CCL19/ CCL21. However, additional evidence is required.

Because fibroblasts are the principal cell type in LT and are closely associated with ligament ossification in AS patients [22], we examined whether CCL19/CCL21 exert an effect on LT fibroblast proliferation or ossification, both of which are reported in AS. Previous studies have demonstrated the various proliferation effects of CCL19/CCL21 on human M cells, CD4 and CD8 T cells, bone marrow and cord blood CD34+ cells [15,27,29-31]. A recent study reported a minor role of CCL19/CCL21 on the survival, proliferation or migration of adult neural precursor cells [32]. Alternatively, fibroblasts from various organs displayed enhanced or inhibited proliferation in response to stimulation, such as hypoxia, Beta2-adrenergic
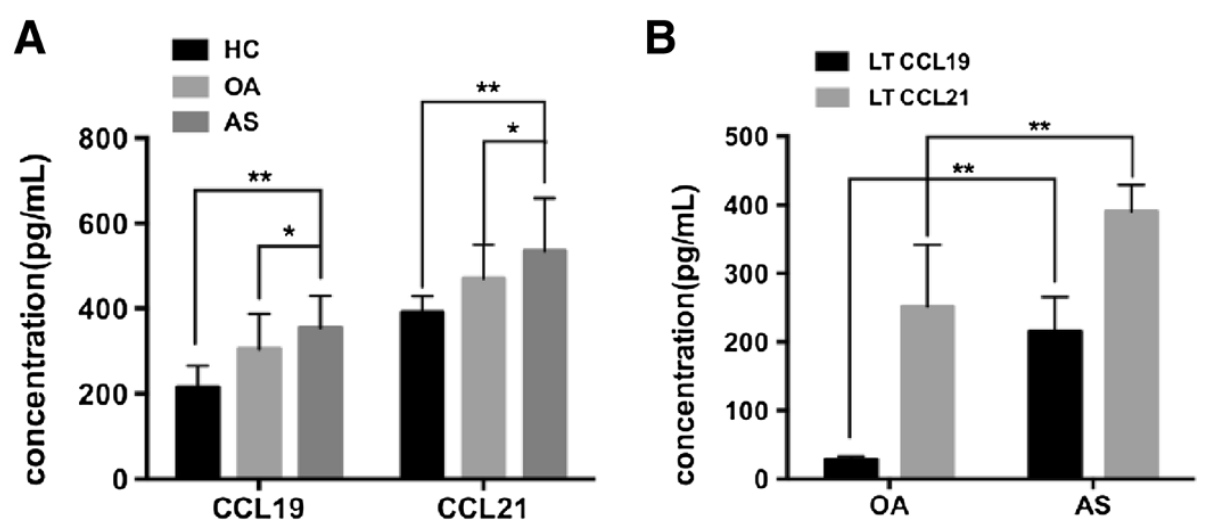

Figure 4 CCL19 and CCL21 levels in serum and ligament tissue (LT). (A) Serum CCL19/CCL21 levels in AS ( $n=42)$, OA $(n=29)$, and healthy controls $(H C, n=16)$. The CCL19 level in the AS group was clearly higher than that in the OA and HC groups (AS vs OA, P=0.011 and AS vs HC, $P=0.003$ ). The $C C L 21$ level displayed a similar distribution (AS vs OA, $P=0.039$ and $A S$ vs HC, $P=0.0075$ ). (B) The levels of CCL19/CCL21 in AS $(n=27)$ and OA $(n=12)$ ligament tissue. The CCL19 level was significantly increased in AS ligament tissue compared to OA LT (AS vS OA, $P=0.024$ ), and $C C L 21$ level displayed a similar difference (AS vs OA, $P=0.041$ ).*P $<0.05$; ${ }^{*} P<0.01$. 
Table 3 CCL19 and CCL21 levels in AS $(n=42)$ serum and correlation to clinical characteristics

\begin{tabular}{llllll}
\hline & \multicolumn{2}{c}{ Serum CCL19 } & & \multicolumn{2}{c}{ Serum CCL21 } \\
\cline { 2 - 3 } \cline { 6 - 7 } & $\mathbf{r}$ & P value & & $\mathbf{r}$ & P value \\
\hline Serum CCL19 level & NA & NA & & 0.546 & $\mathbf{0 . 0 0 3}$ \\
Serum CCL21 level & 0.546 & $\mathbf{0 . 0 0 3}$ & & NA & NA \\
Age & 0.167 & 0.291 & & 0.152 & 0.337 \\
ESR & -0.036 & 0.820 & & 0.062 & 0.698 \\
CRP & 0.191 & 0.226 & & 0.226 & 0.150 \\
BASDAI & 0.233 & 0.137 & 0.182 & 0.250 \\
BASFI & 0.245 & 0.118 & 0.185 & 0.241 \\
BASG & 0.234 & 0.136 & & 0.284 & 0.069 \\
VAS & 0.327 & $\mathbf{0 . 0 3 5}$ & 0.181 & 0.252 \\
Staff time & 0.102 & 0.520 & 0.251 & 0.109 \\
Disease duration & 0.316 & $\mathbf{0 . 0 4 1}$ & 0.254 & 0.105 \\
\hline
\end{tabular}

Abbreviations are the same as those referred to above. Bold values means $\mathrm{P}<0.05$.

receptor agonist treatment and EGF treatment [4,33,34]. Here, we detected no apparent proliferative effect of CCL19/CCL21 on ligament fibroblasts in the $0-200 \mathrm{ng} / \mathrm{ml}$ concentration range. The reason for this result may be that ligament fibroblasts are terminally differentiated cells with limited proliferation capability.

Syndesmophytes are evident in AS and, ligament fibroblasts are involved in their formation [22]. Many investigators have identified the ossification tendency of fibroblasts in LT. Cytokines such as CCL3 has been found to inhibit osteogenesis in myeloma [35]. In this study, we found higher expression of bone markers, such as ALP and OCN, and the transcription factors Runx-2 and osterix after stimulation of AS fibroblasts with CCL19/CCL21. Runx-2 and osterix are key regulators of osteoblast maturation and differentiation and modulate the expression of bone markers, including ALP, COL1, IBSP and OCN [36]. Our results provide an innovative role of CCL19/CCL21 in fibroblast ossification, thus presenting new research targets for ligament lesions in AS. However, the precise mechanism by which CCL19/CCL21 affect mineralization via Runx-2 or osterix is not certain. In RA synovial fibroblasts, CCL19 expression is primarily modulated by lipopolysaccharide (LPS), tumor necrosis factor- $\alpha$ (TNF- $\alpha$ ), interleukin- 1 beta (IL-1 $\beta$ ) and IL-8, whereas CCL21 is modulated by TNF- $\alpha$. Exogenous CCL19/CCL21 treatment up-regulated the expression of VEGF and Ang-I, both of which are crucial mediators of angiogenesis [16]. Parallel studies of RA and OA fibroblasts have also found that CCL19/CCL21 mediated migration [37]. In our study, we detected significantly increased VEGF mRNA expression following CCL19/CCL21 stimulation (data not shown). Ossification and angiogenesis are closely correlated, and VEGF is an essential mediator of both of these processes [38]. Furthermore, VEGF is also regulated by osterix [39]. Based on the above findings, we speculate that inflammatory factors, such as LPS or TNF- $\alpha$, may be regulate CCL19/CCL21, and CCL19/ CCL21 promote fibroblast ossification via the Runx-2osterix-VEGF pathway. However, further evidence is required to validate this hypothesis, and additional studies are necessary.

In this study, we detected higher levels of serum CCL19/CCL21 in both AS and OA patients compared to HC. CCL19/CCL21 were reported to predict the inflammatory state in some diseases $[19,20]$, but we did not detect any correlation between CCL19/CCL21 expression and inflammation or functional characteristics in AS patients. The serum CCL19/CCL21 levels are closely associated, which might indicate a combined effect of pathogenicity. Furthermore, the CCL19 levels correlated to two disease-related global and activity indicators: the VAS score and the disease duration. The VAS score reflects the pain level, and the disease duration may reflect the onset of disease. This result indicates that CCL19 is a relative indicator of early onset in AS patients.

There are some limitations to our study. First, pathological studies demonstrated that multiple cell types, including fibroblasts, periosteal cells, endothelial cells and stem cells, are present in LT, so other cells that express CCR7 should also be considered. Second, all of the patients in our study were admitted for joint replacement surgery and exhibited severely restricted hip activity and a poor BASFI score (average BASFI $=62.24$, indicating severe functional limitations), and these patients do not represent the entire AS population. Third, there is a significant difference in the average age between the AS and OA groups, which may influence the results. Fourth, the precise concentrations of CCL19/CCL21 in AS LT $(n=10)$ and peripheral blood are similar to those of RA synovium and peripheral blood [16], but ossification is not observed in RA patients. Two reasons may account for these results: ossification is caused by changes in the gene expression level in AS, and CCL19/ CCL21 may accelerate this progress; and the concentration of CCL19/CCL21 used in this study, $10 \mathrm{ng} / \mathrm{ml}$, which is far more than the levels in LT, induced the activation of ossification in cultured fibroblasts in vitro. In addition, the number of AS patients included in our study is small for correlation analysis. Further investigation using larger sample sizes would provide more convincing results.

In summary, we observed higher expression of CCL19/CCL21 in AS LT. The increased CCL19/CCL21 displayed pro-ossification potential but did not affect the proliferation of AS ligament fibroblasts. Moreover, the serum CCL19 levels significantly correlated to the serum CCL21 levels, the VAS score and the disease duration, 
which reveals its important role in global disease assessment of AS patients.

\section{Conclusion}

CCL19/CCL21, two chemokine that were higher expressed in AS LT and showed a pro-ossification potential, were also elevated and closed associated in serum samples of AS patients.

\section{Abbreviations}

CCL19: Chemokine (C-C motif) ligand 19; CCL21: Chemokine (C-C motif) ligand 21; AS: Ankylosing spondylitis; LT: Ligament tissue; CCR7: Chemokine (C-C motif) receptor 7; OA: Osteoarthritis; ALP: Alkaline phosphatase; OCN: Osteocalcin; COL1: Collagenase I; IBSP: Integrin-binding sialoprotein; Runx-2: Runt-related transcription factor-2; HO: Heterotopic ossification; RA: Rheumatoid arthritis; ESR: Erythrocyte sedimentation rate; CRP: C-reactive protein; BASDAl: Bath Ankylosing Spondylitis Disease Activity Index; BASFI: Bath Ankylosing Spondylitis Functional Index; VAS: Visual analog scale; BAS-G: Bath Ankylosing Spondylitis Patient Global Score.

\section{Competing interests}

The authors declare that they have no competing interests.

\section{Authors' contributions}

YQ conceived and designed the study, prepared the manuscript and performed the real-time PCR and IHC analyses; LD collected the clinical and laboratory data and the serum and tissue samples and performed cell culture, the CCK8 assay and ELISA; ZJ processed the data and interpreted and plotted the results. TWW and ZYM provides great help to review, selection and collection of patient data. All authors edited the manuscript and read and approved the final manuscript for publication.

\section{Author's information}

Common communication: Miao Min Yong.

\section{Acknowledgments}

This study was supported by the Shanghai Key Basic Research Project (10JC1417900), and National Natural Science Foundation of China (NTFC) (31070747).

\section{Author details}

'Department of Orthopedics, Changhai Hospital Affiliated to the Second Military Medical University, Changhai Road 168, Shanghai 200433, Yangpu district, P. R. China. ${ }^{2}$ School of Kinesiology, Shanghai University of Sport, Hengren Road 200, Shanghai 200438, Yangpu District, P. R. China.

${ }^{3}$ Department of Biochemistry and Molecular Biology, Second Military Medical University, Xiangyin Road 800, Shanghai 200433, Yangpu District, P. R. China.

Received: 30 June 2014 Accepted: 27 August 2014

Published: 26 September 2014

\section{References}

1. Brophy S, Cooksey R, Davies H, Dennis MS, Zhou SM, Siebert S: The effect of physical activity and motivation on function in ankylosing spondylitis: a cohort study. Semin Arthritis Rheum 2013, 42(6):619-626.

2. Vander Cruyssen B, Vastesaeger N, Collantes-Estevez E: Hip disease in ankylosing spondylitis. Curr Opin Rheumatol 2013, 25(4):448-454.

3. Hamdi W, Chelli-Bouaziz M, Ahmed MS, Ghannouchi MM, Kaffel D, Ladeb MF, Kchir MM: Correlations among clinical, radiographic, and sonographic scores for enthesitis in ankylosing spondylitis. Joint Bone Spine 2011, 78(3):270-274.

4. Zhang HY, Liu R, Xing YJ, Xu P, Li Y, Li CJ: Effects of hypoxia on the proliferation, mineralization and ultrastructure of human periodontal ligament fibroblasts. Exp Ther Med 2013, 6(6):1553-1559.

5. Yang HS, Lu XH, Chen DY, Yuan W, Yang LL, He HL, Chen Y: Upregulated expression of connexin43 in spinal ligament fibroblasts derived from patients presenting ossification of the posterior longitudinal ligament. Spine 2011, 36(26):2267-2274
6. Yamashita M, Iwama N, Date F, Shibata N, Miki H, Yamauchi K, Sawai T, Sato S, Takahashi T, Ono M: Macrophages participate in lymphangiogenesis in idiopathic diffuse alveolar damage through CCL19-CCR7 signal. Hum Pathol 2009, 40(11):1553-1563.

7. Ekkehard Ziegler MO, Bulfone-Paus S, Förster R, Kunzendorf U, Krautwald S: CCR7 Signaling inhibits T cell proliferation. J Immunol 2007, 179:6485-6493.

8. Luther SA, Tang HL, Hyman PL, Farr AG, Cyster JG: Coexpression of the chemokines ELC and SLC by T zone stromal cells and deletion of the ELC gene in the plt/plt mouse. Proc Natl Acad Sci U S A 2000, 97(23):12694-12699.

9. Beckmann JH, Yan S, Luhrs H, Heid B, Skubich S, Forster R, Hoffmann MW: Prolongation of allograft survival in ccr7-deficient mice. Transplantation 2004, 77(12):1809-1814.

10. Debes GF, Arnold CN, Young AJ, Krautwald S, Lipp M, Hay JB, Butcher EC: Chemokine receptor CCR7 required for T lymphocyte exit from peripheral tissues. Nat Immunol 2005, 6(9):889-894.

11. Luchtefeld M, Grothusen C, Gagalick A, Jagavelu K, Schuett H, Tietge UJ, Pabst O, Grote K, Drexler H, Forster R, Schieffer B: Chemokine receptor 7 knockout attenuates atherosclerotic plaque development. Circulation 2010, 122(16):1621-1628.

12. Zhang Q, Sun L, Yin L, Ming J, Zhang S, Luo W, Qiu X: CCL19/CCR7 upregulates heparanase via specificity protein-1 (Sp1) to promote invasion of cell in lung cancer. Tumour Biol 2013, 34(5):2703-2708.

13. Wilson JL, Burchell J, Grimshaw MJ: Endothelins induce CCR7 expression by breast tumor cells via endothelin receptor $A$ and hypoxia-inducible factor-1. Cancer Res 2006, 66(24):11802-11807.

14. Raman D, Baugher PJ, Thu YM, Richmond A: Role of chemokines in tumor growth. Cancer Lett 2007, 256(2):137-165.

15. Sanchez-Sanchez N, Riol Blanco L, Rodriguez-Fernandez JL: The multiple personalities of the chemokine receptor CCR7 in Dendritic Cells. $J$ Immunol 2006, 176:7. Epub 2006.

16. Pickens SR, Chamberlain ND, Volin MV, Pope RM, Mandelin AM 2nd, Shahrara S: Characterization of CCL19 and CCL21 in rheumatoid arthritis. Arthritis Rheum 2011, 63(4):914-922.

17. Nureki S, Miyazaki E, Ishi T, Ito T, Takenaka R, Ando M, Kumamoto T: Elevated concentrations of CCR7 ligands in patients with eosinophilic pneumonia. Allergy 2013, 1387:1395.

18. Cassier PA, Treilleux I, Bachelot T, Ray-Coquard I, Bendriss-Vermare N, Menetrier-Caux C, Tredan O, Goddard-Leon S, Pin JJ, Mignotte H, Bathélémy-Dubois C, Caux C, Lebecque S, Blay JY: Prognostic value of the expression of C-Chemokine Receptor 6 and 7 and their ligands in non-metastatic breast cancer. BMC Cancer 2011, 11:213.

19. Mathes AL, Christmann RB, Stifano G, Affandi AJ, Radstake TR, Farina GA, Padilla C, McLaughlin S, Lafyatis R: Global chemokine expression in systemic sclerosis (SSC): CCL19 expression correlates with vascular inflammation in SSc skin. Ann Rheum Dis 2013.

20. Sellam J, Rouanet S, Hendel-Chavez H, Miceli-Richard C, Combe B, Sibilia J, Le Loet X, Tebib J, Jourdan R, Dougados M, Taoufik Y, Mariette X: CCL19, a $B$ cell chemokine, is related to the decrease of blood memory B cells and predicts the clinical response to rituximab in patients with rheumatoid arthritis. Arthritis Rheum 2013, 65(9):2253-2261.

21. van der Linden S, Valkenburg HA, Cats A: Evaluation of diagnostic criteria for ankylosing spondylitis. a proposal for modification of the New York criteria. Arthritis Rheum 1984, 27(4):361-368.

22. Yu F, Cui Y, Zhou X, Zhang X, Han J: Osteogenic differentiation of human ligament fibroblasts induced by conditioned medium of osteoclast-like cells. Biosci Trends 2011, 5(2):46-51.

23. Yeh Y, Yang Y, Yuan K: Importance of CD44 in the proliferation and mineralization of periodontal ligament cells. J Periodontal Res 2014 PubMed PMID: 24517578

24. Choi MH, Noh WC, Park JW, Lee JM, Suh JY: Gene expression pattern during osteogenic differentiation of human periodontal ligament cells in vitro. J Periodontal Implant Sci 2011, 41(4):167-175.

25. Asari T, Furukawa K, Tanaka S, Kudo H, Mizukami H, Ono A, Numasawa T, Kumagai G, Motomura S, Yagihashi S, Toh S: Mesenchymal stem cell isolation and characterization from human spinal ligaments. Biochem Biophys Res Commun 2012, 417(4):1193-1199.

26. Song M, Kim H, Choi Y, Kim K, Chung C: Skeletal myogenic differentiation of human periodontal ligament stromal cells isolated from orthodontically extracted premolars. Korean J Orthod 2012, 42(5):249-254. 
27. Wornle M, Schmid H, Merkle M, Banas B: Effects of chemokines on proliferation and apoptosis of human mesangial cells. BMC Nephrol 2004, 5:8.

28. Abe Y, Ohtsuji M, Ohtsuji N, Lin Q, Tsurui H, Nakae S, Shirai T, Sudo K, Hirose S: Ankylosing enthesitis associated with up-regulated IFN-gamma and IL-17 production in (BXSB x NZB) F(1) male mice: a new mouse model. Mod Rheumatol 2009, 19(3):316-322.

29. Broxmeyer HE, Kim CH: SLC/Exodus2/6Ckine/TCA4 induces chemotaxis of hematopoietic progenitor cells: differential activity of ligands of CCR7 CXCR3, or CXCR4 in chemotaxis vs. suppression of progenitor proliferation. J Leukoc Biol 1999, 66:7.

30. Pierce EM, Carpenter K, Jakubzick C, Kunkel SL, Evanoff H, Flaherty KR, Martinez FJ, Toews GB, Hogaboam CM: Idiopathic pulmonary fibrosis fibroblasts migrate and proliferate to CC chemokine ligand 21. Eur Respir J 2007, 29(6):1082-1093.

31. Ploix C, LO D, Carson MJ: A ligand for the chemokine receptor CCR7 can influence the homeostatic proliferation of CD4 T cells and progression of autoimmunity. J Immunol 2001, 167(12):6724-6730.

32. Turbic A, Leong SY, Turnley AM: Chemokines and inflammatory mediators interact to regulate adult murine neural precursor cell proliferation, survival and differentiation. PLoS One 2011, 6(9):e25406.

33. Gruber R, Leimer M, Fischer MB, Agis H: Beta2-adrenergic receptor agonists reduce proliferation but not protein synthesis of periodontal fibroblasts stimulated with platelet-derived growth factor-BB. Arch Oral Biol 2013, 58(12):1812-1817.

34. Kimura H, Okubo N, Chosa N, Kyakumoto S, Kamo M, Miura H, Ishisaki A: EGF positively regulates the proliferation and migration, and negatively regulates the myofibroblast differentiation of periodontal ligament-derived endothelial progenitor cells through MEK/ERK- and JNK-dependent signals. Cell Physiol Biochem 2013, 32(4):899-914.

35. Vallet S, Pozzi S, Patel K, Vaghela N, Fulciniti MT, Veiby P, Hideshima T, Santo L, Cirstea D, Scadden DT, Anderson KC, Raje N: A novel role for CCL3 (MIP-1alpha) in myeloma-induced bone disease via osteocalcin downregulation and inhibition of osteoblast function. Leukemia 2011, 25(7):1174-1181.

36. Zhu J, Shimizu E, Zhang X, Partridge NC, Qin L: EGFR signaling suppresses osteoblast differentiation and inhibits expression of master osteoblastic transcription factors Runx2 and Osterix. J Cell Biochem 2011, 112(7):1749-1760.

37. Bruhl H, Mack M, Niedermeier M, Lochbaum D, Scholmerich J, Straub RH: Functional expression of the chemokine receptor CCR7 on fibroblast-like synoviocytes. Rheumatology 2008, 47(12):1771-1774.

38. Yang $Y Q$, Tan YY, Wong R, Wenden A, Zhang LK, Rabie AB: The role of vascular endothelial growth factor in ossification. Int J Oral Sci 2012, 4(2):64-68.

39. Tang W, Yang F, Li Y, de Crombrugghe B, Jiao H, Xiao G, Zhang C Transcriptional regulation of Vascular Endothelial G Factor (VEGF) by osteoblast-specific transcription factor Osterix (Osx) in osteoblasts. J Biol Chem 2012, 287(3):1671-1678.

doi:10.1186/1471-2474-15-316

Cite this article as: Qin et al:: Increased CCL19 and CCL21 levels promote fibroblast ossification in ankylosing spondylitis hip ligament tissue. BMC Musculoskeletal Disorders 2014 15:316.

\section{Submit your next manuscript to BioMed Central and take full advantage of:}

- Convenient online submission

- Thorough peer review

- No space constraints or color figure charges

- Immediate publication on acceptance

- Inclusion in PubMed, CAS, Scopus and Google Scholar

- Research which is freely available for redistribution 